\title{
A case of indolent endocarditis
}

\author{
Hanane Benbarkat $M D^{1}$, Karima Addetia $M D^{1}$, Amir H Salehi MD², Donald Sheppard MD ${ }^{1}$, Thao Huynh MD ${ }^{1}$
}

\section{CASE PRESENTATION}

69-year-old man with aortic stenosis underwent coronary artery Abypass grafting and bioprosthetic aortic valve replacement (AVR) in November 2007. His medical history was remarkable for a polypectomy in 2007, gout and chronic kidney disease (creatinine level at presentation $150 \mu \mathrm{mol} / \mathrm{L})$.

In September 2008, the patient presented to the hospital with fever and a right middle cerebral artery infarct. His neurological symptoms ultimately resolved. An echocardiogram demonstrated vegetation on the aortic valve bioprosthesis with a periaortic abscess. Blood cultures grew Staphylococcus epidermidis. An increase in the size of the periaortic abscess one month later, despite appropriate intravenous antibiotic therapy, required another AVR (Carpentier Edwards Magna 23 bioprosthesis, Edwards Lifesciences LLC, USA). He was then treated with antibiotics for an additional six weeks. The prosthesis culture grew Penicillium species 11 days after his surgery, and valve pathology showed fungal hyphae one month later (Figure 1). The patient was asymptomatic. He was offered amphotericin B treatment but refused.

He remained asymptomatic until May 2010 when he presented with a two-week history of exertional dyspnea. He developed a cold right leg two days before this presentation. On examination, he was afebrile with normal blood pressure and heart rate. Chest examination was unremarkable. Cardiovascular examination revealed a 2/6 systolic ejection murmur at the left second intercostal space with radiation to the right supraclavicular space. There was no diastolic murmur. The jugular venous pressure was normal. The right leg was cold and peripheral pulses were nonpalpable. Laboratory investigations showed a normal white blood cell count, an erythrocyte sedimentation rate of $82 \mathrm{~mm} / \mathrm{h}$ and his chest $\mathrm{x}$-ray was normal. The PR interval on the electrocardiogram was $280 \mathrm{~ms}$ compared with $200 \mathrm{~ms}$ in October 2008. A repeat echocardiogram demonstrated a $27 \mathrm{~mm} \times 15 \mathrm{~mm}$ vegetation on the aortic bioprosthesis with a periaortic mass highly suggestive of an aortic root abscess. The mean gradient across the aortic valve had increased to $43 \mathrm{mmHg}$ from $14 \mathrm{mmHg}$ in June 2009. The patient was empirically treated for prosthetic valve endocarditis with vancomycin, gentamycin and rifampin.

\section{DIAGNOSIS}

Eight days after admission, blood cultures were positive for fungal hyphae, and empirical therapy with caspofungin was begun. The organism was identified as Paecilomyces lilacinus. An ophthalmological evaluation demonstrated right fungal endophthalmitis. Sensitivity testing using microbroth dilution methods was performed and yielded a minimal inhibitory concentration (MIC)/minimum effective concentration (MEC) of: amphotericin B $1 \mu \mathrm{g} / \mathrm{mL}$; posaconazole $0.25 \mu \mathrm{g} / \mathrm{mL}$; voriconazole $1 \mu \mathrm{g} / \mathrm{mL}$; and caspofungin $>256 \mu \mathrm{g} / \mathrm{mL}$. Casopofungin was discontinued and posaconazole $200 \mathrm{mg}$ orally four times daily was initiated. Blood cultures during posaconazole therapy remained negative after seven days of incubation, and the patient was discharged home after declining valve replacement.

Eleven days after initiation of posaconazole therapy, the patient presented again with a cold left leg, and an emergent thrombectomy was performed. Pathology was consistent with thrombus containing filamentous fungi. Liposomal amphotericin B (3 $\mathrm{mg} / \mathrm{kg} /$ day) was

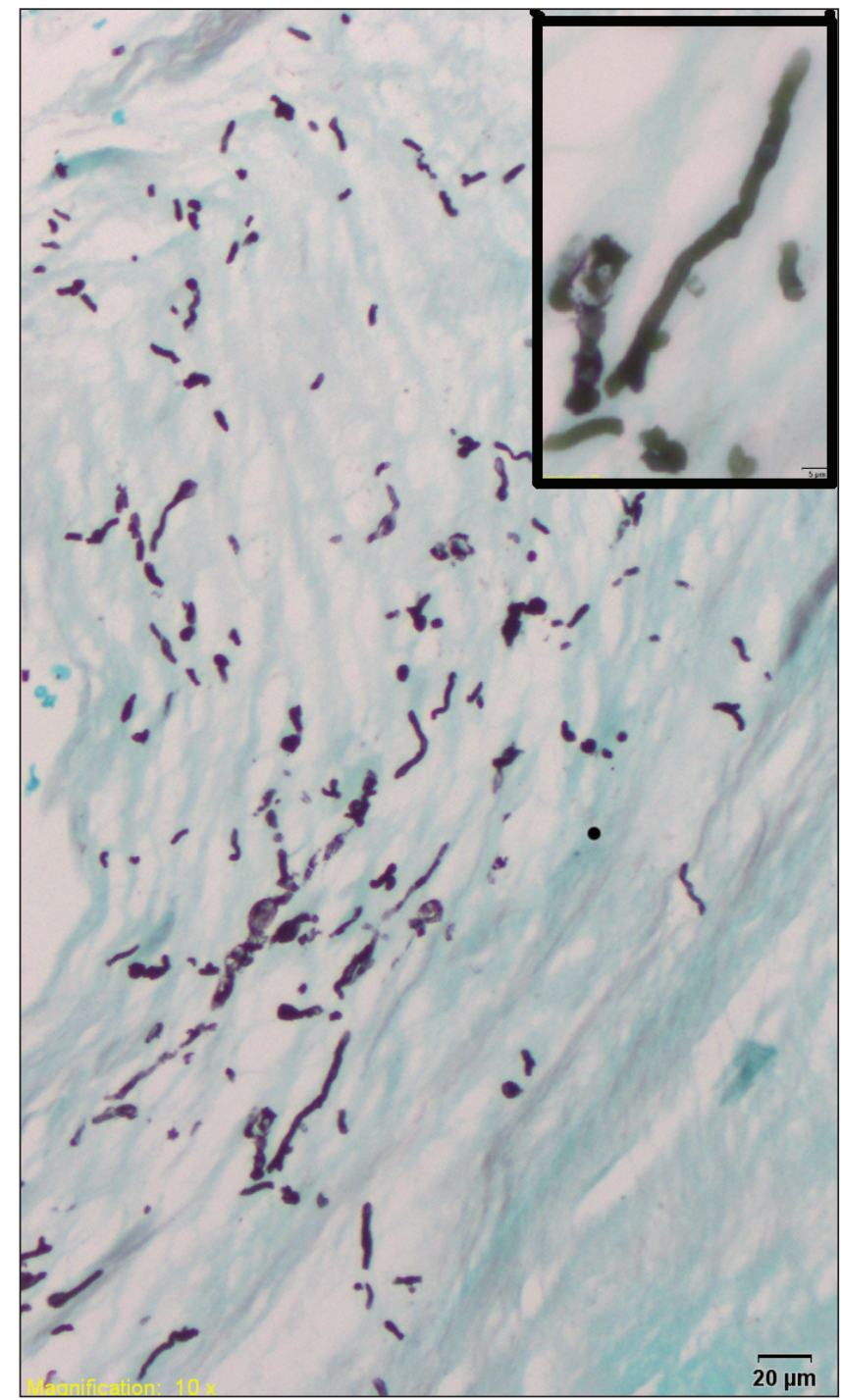

Figure 1) Microscopic section of the aortic valve resected in 2008. Silver stain revealing branching fungal hyphae, with possible septation visualized on higher magnification (right upper panel)

added to posaconazole for synergism. Blood cultures were positive for Paecilomyces species. A transesophageal echocardiogram showed moderate perivalvular aortic regurgitation. A decision was made to replace the aortic valve.

Postoperative fungal cultures were negative. Valve pathology showed adherent thrombus with extensive infiltration by fungal hyphae. Four months after surgery, the patient was discharged on lifelong voriconazole therapy.

${ }^{1}$ Department of Medicine; ${ }^{2}$ Department of Pathology, McGill University, Montreal, Quebec

Correspondence: Dr Hanane Benbarkat, 687 Pine Avenue, Room 10.02, Montreal, Quebec H3A 1A1.

Telephone 514-843-1506, fax 514-843-1725, e-mail hanane.benbarkat@mail.mcgill.ca 


\section{DISCUSSION}

Prosthetic valve endocarditis represents $20 \%$ to $30 \%$ of all cases of infective endocarditis. It can be classified as early ( $\leq 1$ year after surgery) or late ( $>1$ year). Pathogens causing late prosthetic valve endocarditis are usually the same as those causing native valve endocarditis (1).

We report a case involving a patient with an 18-month history of untreated indolent $P$ lilacinus endocarditis after bioprosthetic AVR. This is the second case of $P$ lilacinus endocarditis reported in the literature (2) and is remarkable for the duration of infection and its indolent course. All other cases of Paecilomyces endocarditis were secondary to Paecilomyces variotti.

The prosthesis culture in 2008 grew Penicillium species, which were originally believed to be a contaminant. Upon review of the culture description, this organism was almost certainly a Paecilomyces species, because Paecilomyces species and Penicillium species are closely related morphologically (3). Valve pathology in 2008 revealed Gram-positive cocci and fungal hyphae, strongly suggesting that Paecilomyces species were present at that time (Figure 1). We were not able to confirm this hypothesis because the sample was no longer available; however, the development of a second filamentous fungal endocarditis in the same patient seems implausible.

$P$ lilacinus and $P$ variotti are the two species most frequently associated with human diseases (4). P lilacinus infects both immunocompromised and immunocompetent hosts. The portal of entry could be via breakdown of the skin barrier, inhalation or through the introduction of a foreign body (eg, prosthetic cardiac valve and peritoneal catheters). P lilacinus most commonly causes cutaneous, subcutaneous and ocular infections (4).

It is important to differentiate between Paecilomyces species, because $P$ lilacinus and $P$ variotti have very different antifungal properties. $P$ variotti is susceptible to amphotericin $B$ whereas $P$ lilacinus is intrinsically resistant to conventional antifungals (5). Posaconazole is the agent with the lowest MIC when compared with other triazoles (MIC from $0.12 \mathrm{mg} / \mathrm{L}$ to $0.5 \mathrm{mg} / \mathrm{L}$ ). In vitro activity of echinocandins is controversial. Our patient was treated with posaconazole, which appeared to be the most promising therapeutic option $(4,6)$. However, after 11 days of treatment, he presented with an embolic event and positive blood cultures. In light of the MIC testing results, we elected to add liposomal amphotericin B as combination therapy.
In vitro synergism has been observed for some antifungal combinations, without any clinical significance for these observations (7). In our case, sterilization was not achieved after 26 days of antifungal therapy, including 15 days of combination therapy (posoconazole and amphotericin B). Removal of the infected valve was essential for the resolution of the infection. The patient was discharged on voriconazole because the therapeutic troughs achieved for this drug are almost 10-fold higher compared with posaconazole, a fact likely to be relevant in the treatment of endocarditis $(8,9)$. Moreover, the development of an embolic event to his leg while on posaconazole was another reason for favouring voriconazole.

This is the first reported case of indolent $P$ lilacinus endocarditis. The present case highlights the implications of identifying hyphae on valve pathology and the difficulty in diagnosing and treating fungal endocarditis.

\section{REFERENCES}

1. Nataloni M, Pergolini M, Rescigno G, et al. Prosthetic valve endocarditis. J Cardiovasc Med 2010;11:869-83.

2. Muller H, Cikirikcioglu M, Lerch R. Subaortic aneurysm caused by Paecilomyces lilacinus endocarditis. Arch Cardiovasc Dis 2008;101:803-4.

3. The Johns Hopkins microbiology newsletter, Vol 25, No 17. <www. hopkinsmedicine.org/bin/n/h/Vol25No17 Paecilomyces.doc $>$. (Accessed July 24, 2010).

4. Pastor FJ, Guarro J. Clinical manifestations, treatment and outcome of Paecilomyces lilacinus infections. Clin Microbiol Infect 2006;12:948-60.

5. Castelli MV, Alastruey-Izquierdo A, Cuesta I, et al. Susceptibility testing and molecular classification of Paecilomyces spp. Antimicrob Agents Chemother 2008;52:2926-8.

6. Rodriguez MM, Pastor FJ, Serena C, et al. Posaconazole efficacy in a murine disseminated infection caused by Paecilomyces lilacinus. J Antimicrob Chemoth 2009;63:361-4.

7. Ortoneda M, Capilla J, Pastor FJ, et al. In vitro interactions of approved and novel drugs against Paecilomyces spp. Antimicrob Agents Chemother 2004:48:2727-9.

8. Pascual A, Calandra T, Bolay S, et al. Voriconazole therapeutic drug monitoring in patients with invasive mycoses improves efficacy and safety outcomes. J Clin Infect Dis 2008;46:201-11.

9. Jang SH, Colangelo PM, Gobburu JVS. Exposure-response of posaconazole used for prophylaxis against invasive fungal infections: Evaluating the need to adjust doses based on drug concentrations in plasma. Clin Pharmacol Ther 2009:88:115-19. 


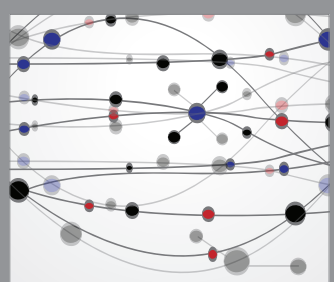

The Scientific World Journal
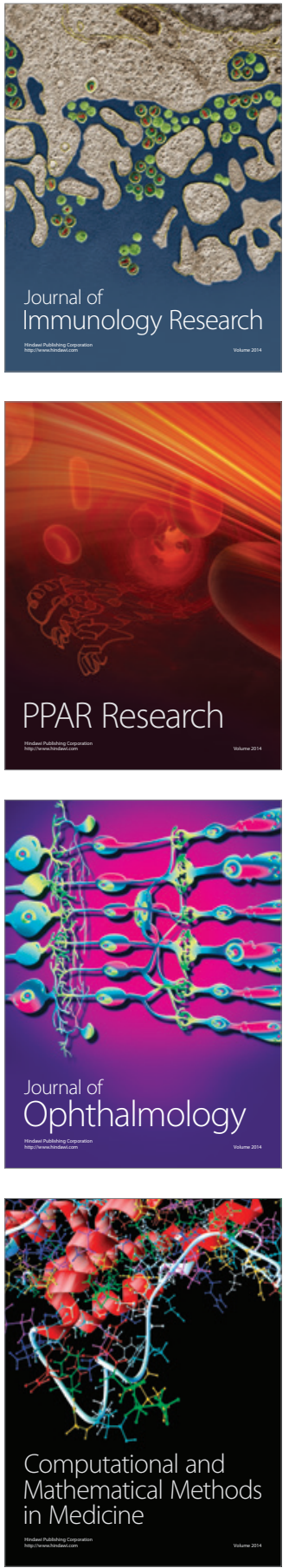

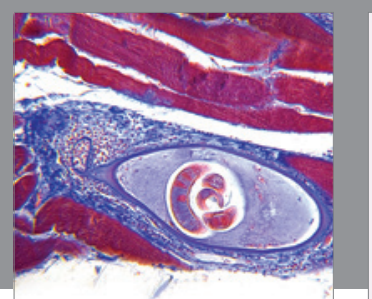

Gastroenterology Research and Practice

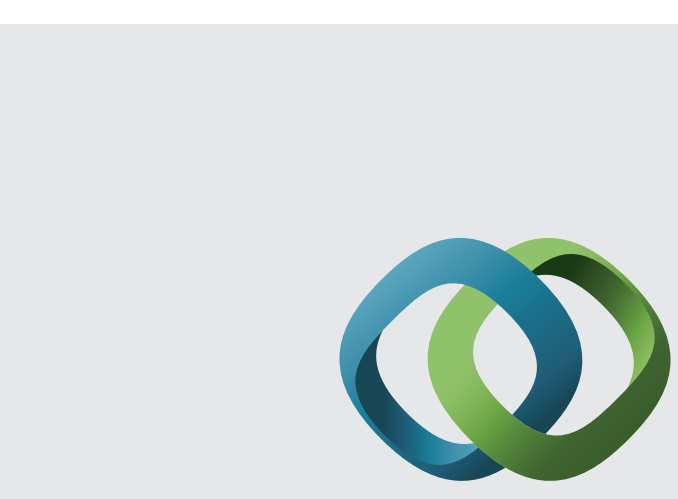

\section{Hindawi}

Submit your manuscripts at

http://www.hindawi.com
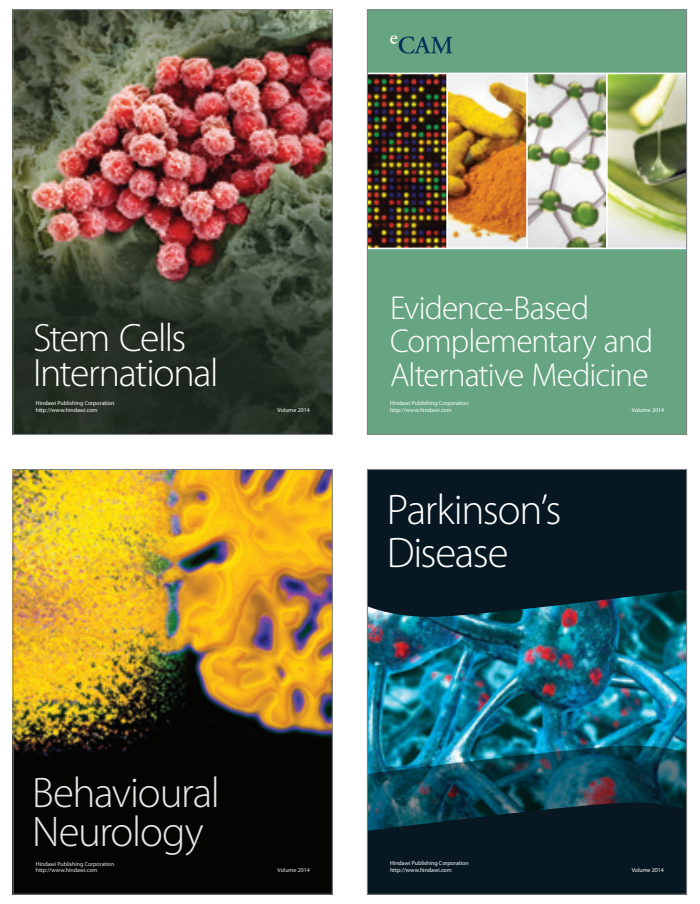
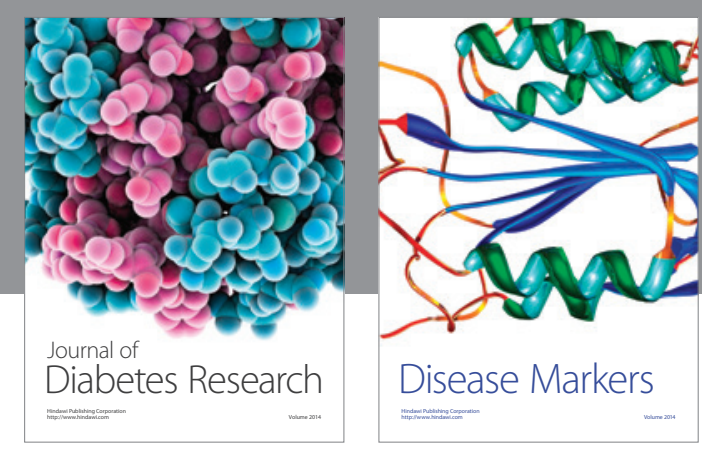

Disease Markers
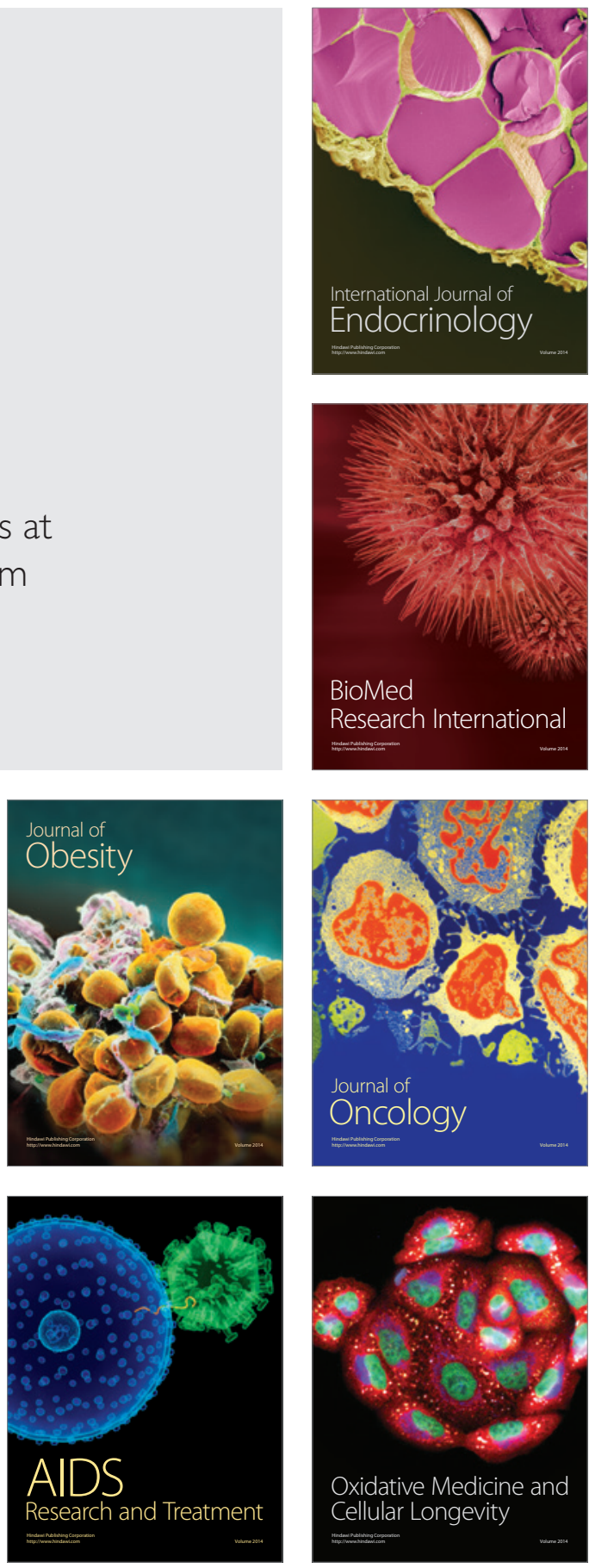